This work reports the piezoresistance of silicon nanowires fabricated using focused ion beam and wet etching for NEMS mechanical sensors.
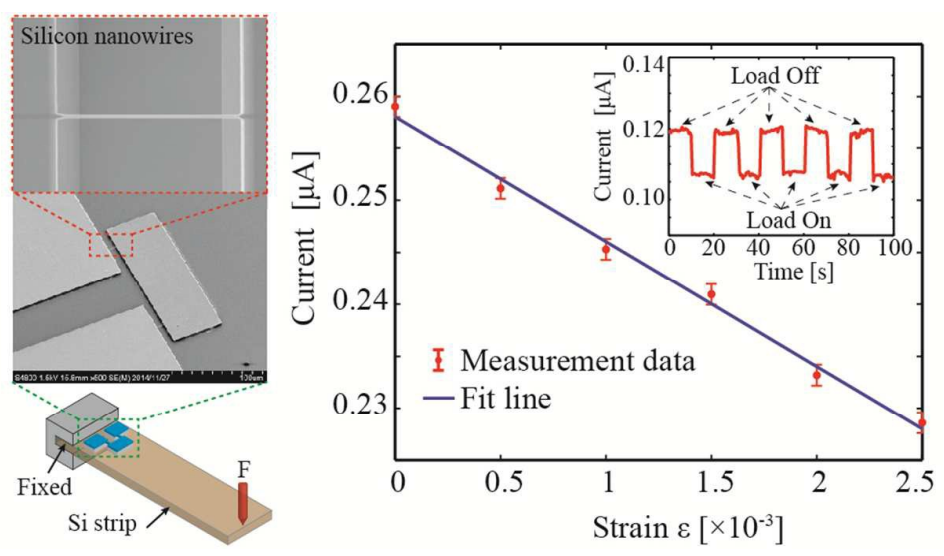


\title{
Piezoresistive effect of p-type silicon nanowires fabricated by a top- down process using FIB implantation and wet etching
}

\author{
Hoang-Phuong Phan, ${ }^{a}$ Takahiro Kozeki, $^{c}$ Toan Dinh, ${ }^{a}$ Tatsuya Fujii, ${ }^{c}$ Afzaal Qamar, ${ }^{a}$ Yong Zhu, ${ }^{a}$ \\ Takahiro Namazu, ${ }^{c}$ Nam-Trung Nguyen, ${ }^{a}$ and Dzung Viet Dao ${ }^{a, b}$
}

Received * * 2015

DOI: 10.1039/b000000x

The piezoresistive effect in silicon nanowires (SiNWs) has attracted a great deal of interest for NEMS devices. Most of the piezoresistive SiNWs reported in the literature were fabricated using the bottom up method or top down processes such as electron beam lithography (EBL). Focused ion beam (FIB), on the other hand, is more compatible with CMOS integration than the bottom up method, and is simpler and more capable of fabricating very narrow $\mathrm{Si}$ nanostructures compared to EBL and photolithography. Taking the advantages of FIB, this paper presents for the first time the piezoresistive effect of p-type SiNWs fabricated using focused ion beam implantation and wet etching. The SiNWs were locally amorphized by $\mathrm{Ga}^{+}$ion implantation, selectively wet-etched, and thermally annealed at $700^{\circ} \mathrm{C}$. A relatively large gauge factor of approximately 47 was found in the annealed SiNWs, indicating the potential of using the piezoresistive effect in top-down fabricated SiNWs for developing NEMS sensors.

Discovered by Smith in 1954, the piezoresistive effect in semiconductors has been investigated and employed in various Micro Electromechanical Systems (MEMS) applications ${ }^{1-6}$. Thanks to its availability, advanced MEMS fabrication process, and large gauge factor, silicon ( $\mathrm{Si}$ ) is among the first choice materials for developing piezoresistive transducers. The piezoresistive effect of bulk silicon has been utilized in AFM (atomic force microscope) cantilevers ${ }^{7}$, acceleration, tactile, and pressure sensors ${ }^{8-10}$.

Recent research has paid a great attention to the piezoresistive effect of silicon nano structures, such as nanowires and nanosheets, to elucidate the quantum confinement effect in enhancement of the stress/strain sensing property for highly sensitive and miniaturized sensing devices ${ }^{11,12}$. The giant piezoresistive effect in $\mathrm{Si}$ nanowires ( $\mathrm{SiNWs)}$ with a gauge factor of up to 5000 reported by He and Yang has been a great motivation for a large number of studies on the characterization of the nanoscale effect in piezoresistance ${ }^{13,14}$. Lungstein et al. reported the piezoresistive effect of ultra-strained

\footnotetext{
* Email of corresponding author: hoangphuong.phan@griffithuni.edu.au

a Queensland Micro- and Nanotechnology Centre, Griffith University, Queensland, Australia.

${ }^{b}$ School of Engineering, Griffith University, Queensland, Australia.

${ }^{c}$ Department of Mechanical Engineering, University of Hyogo, Hyogo, Japan
}

SiNWs grown by the vapor-liquid-solid method, with a gauge factor comparable to that of bulk $\mathrm{Si}^{15}$. Zhang et al. characterized the influence of strain on electrical conductance of SiNWs grown by a bottom up method, and reported a gauge factor of $67.1^{16}$. The piezoresistance of SiNWs reported in the literature shows different gauge factor values, varying between several tens to several thousands ${ }^{17-20}$. This large deviation of the gauge factors indicates that the piezoresistive effect in Si nanowires significantly depends on the surface state, concentration (e.g. depleted and highly doped Si), and the fabrication process of Si nanostructures. Additionally, the controversy regarding the existence of the giant piezoresistive effect in Si nanowires implies that more studies need to be carried out to better understand this effect in nanoscale $\mathrm{Si}^{11}$.

As well as the work on bottom-up grown $\mathrm{Si}$, several studies have been carried out on the top-down fabricated SiNWs. Compared to the bottom-up process, the top-down process is more compatible with the conventional MEMS fabrication and CMOS integration. In most of the previous studies, SiNWs were fabricated using the electron beam lithography (EBL) or photolithography process in which a photoresist layer exposured to electron-beams or ultraviolet-light was employed as the etching mask to form SiNWs underneath ${ }^{18,20,21}$. In comparison to these processes, focused ion beam (FIB) is simpler and has become increasingly popular in the fabrication of $\mathrm{Si}$ nanostructures $^{23}$. Additionally, FIB could also be utilized in device modifications because it can be used before, after or as a step in any lithography process ${ }^{24}$. Furthermore, the thickness of SiNWs fabricated by FIB can also be accurately controlled by the penetration depth of the implanted ion, while the other top down processes (e.g. EBL, photolithography) require additional time-consuming steps such as wet/dry etching Si or thermal oxidation ${ }^{25}$.

To the best of our knowledge, to date, there has been no report on the characterization of the piezoresistive effect of SiNWs fabricated by localized amorphization through FIB implantation, selective wet etching, and re-crystallization by thermal annealing process. As the properties of SiNWs are changed due to amorphization and post-FIB thermal treatment $^{26}$, the piezoresistive effect of Si NWs fabricated using this process is expected to be different from that of bulk single crystalline $\mathrm{Si}$. In this paper, we report on the piezoresistive effect in released SiNWs fabricated by a top-down process using 
the focused ion beam (FIB) method followed by wet etching of $\mathrm{Si}$ and thermal annealing. A relatively large gauge factor found in this top-down fabricated SiNWs indicates its high potential for nano electro mechanical systems (NEMS).

The SiNWs were fabricated from a silicon-on-insulator (SOI) wafer with the thicknesses of the device layer (lowdoped n-type $\mathrm{Si}$ with a carrier concentration below $10^{14}$ $\mathrm{cm}^{-3}$ ), the insulation layer and the substrate layer being 2 $\mu \mathrm{m}, 0.2 \mu \mathrm{m}$ and $200 \mu \mathrm{m}$, respectively. In the first step, the SOI wafer was diced into smaller strips with a dimension of $200 \mu \mathrm{m} \times 3 \mathrm{~mm} \times 20 \mathrm{~mm}$ for the bending experiment. Next, an amorphous Si nanowires embedded in the top of the SOI wafer was formed using gallium ions $\left(\mathrm{Ga}^{+}\right)$implantation (FIB, TMHitachi FB2200) at a dose of approximately $7 \times 10^{15} \mathrm{ions} / \mathrm{cm}^{2}$. Since the etching rate of amorphous $\mathrm{Si}$ is much lower than that of single crystalline $\mathrm{Si}$, the amorphous SiNWs were then formed after under-etching the crystalline $\mathrm{Si}$ layer underneath using wet etching with TMAH (tetramethylammonium hydroxide) as the etchant. Two electrode pads with a dimension of $200 \mu \mathrm{m} \times 200 \mu \mathrm{m}$ were also formed for the electrical measurement. Finally, the amorphous SiNWs were thermally annealed at a high temperature of $700^{\circ} \mathrm{C}$ for 60 minutes in a high vacuum chamber. Figure 1 shows the scanning electron microscope (SEM) images of the fabricated SiNWs with their length of $10 \mu \mathrm{m}$, thickness of $60 \mathrm{~nm}$ and widths of 200 and $300 \mathrm{~nm}$.

The amorphization by ion implantation and the recrystallization by thermal annealing of Si were confirmed using optical measurements. Figure 2 (a) shows the cross-sectional high resolution transmission electron microscopy (HRTEM) image of the $\mathrm{Ga}^{+}$implanted Si films, illustrating the amorphized Si layer and the single crystalline Si layer underneath. After being annealed at $700^{\circ} \mathrm{C}$ for 1 hour, the amorphized $\mathrm{Si}$

\section{(a) Fabrication process}
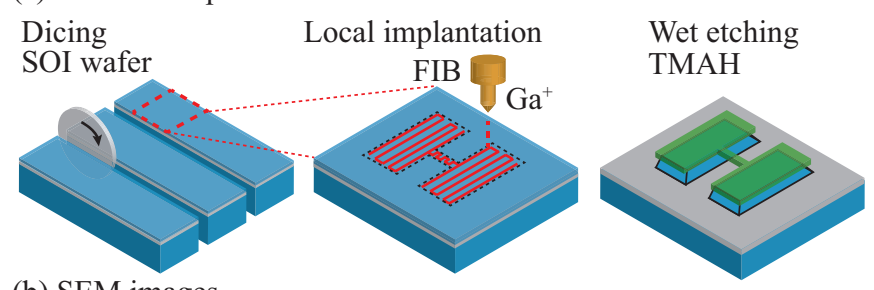

(b) SEM images

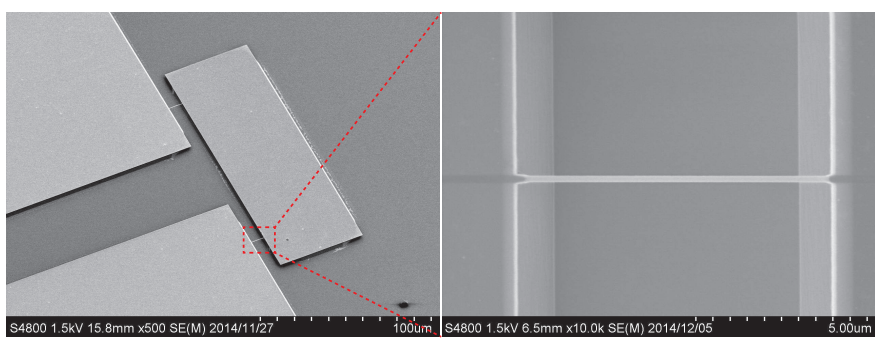

Fig. 1 (a) Fabrication process using $\mathrm{FIB} \mathrm{Ga}^{+}$implantation and wet etching. (b) SEM images of SiNWs.

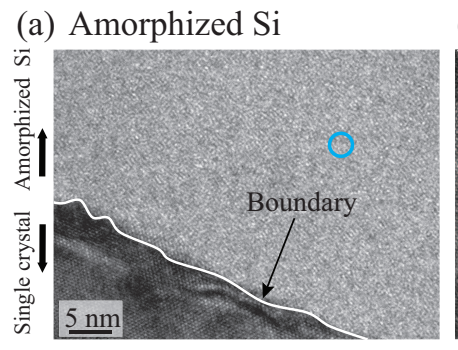

(b) Thermally annealed Si

(d)

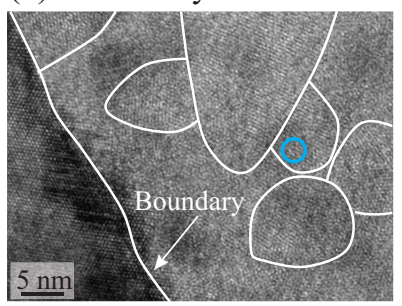

(e)
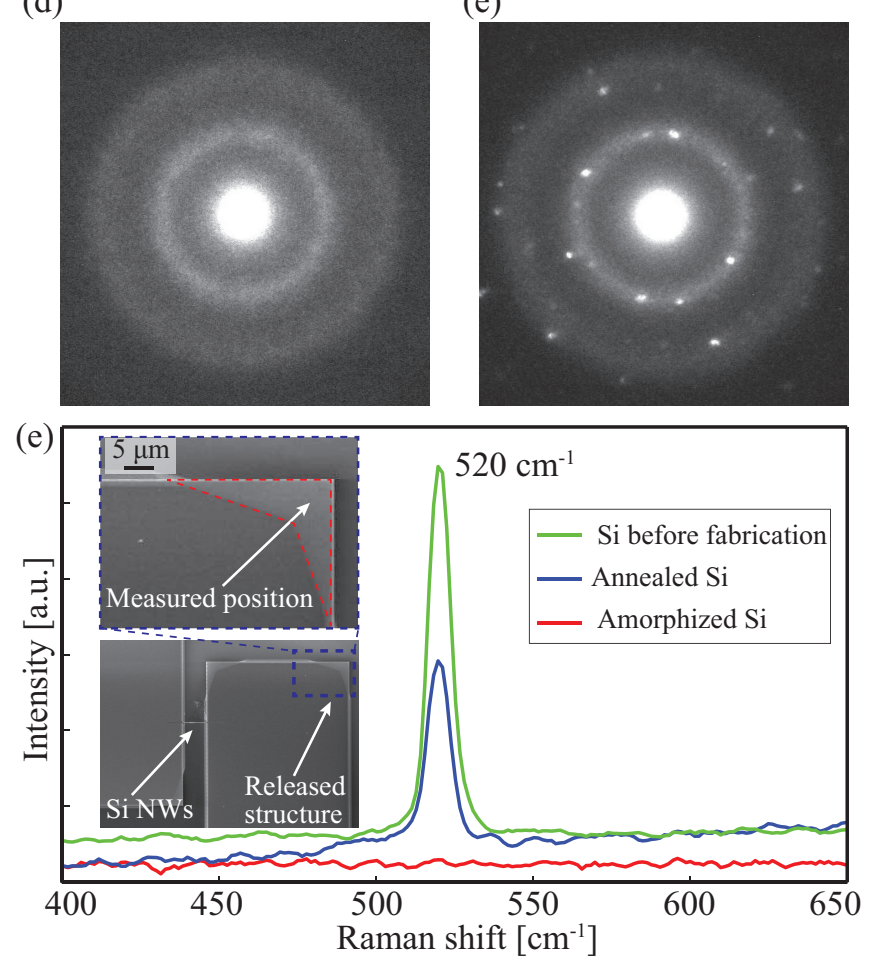

Fig. 2 (a)(b) HRTEM images of ion implanted, and thermally annealed Si, respectively; (c)(d) SAED measurements of the implanted and thermally annealed Si films, respectively. Note that the SEAD data was obtained at the points marked inside the blue circles of figure a and b; (e) Raman spectroscopy of the original Si film, implanted $\mathrm{Si}$ and annealed Si. Note that to reduce the influence of the substrate, the Raman scattering measurements (TMRenishaw inVia Raman Microscope $514 \mathrm{~nm}$ ) were performed on the released $\mathrm{Si}$ frames formed by under-etching the Si substrate as shown in the inset. The laser power in all experiments was $159 \mu \mathrm{W}$.

layer was recrystallized and poly crystalline $\mathrm{Si}$ was observed under HRTEM, Fig. 2 (b). The grain size of the recrystallized Si layer was estimated based on the HRTEM image using an image processing technique ${ }^{27}$. Accordingly, the annealed poly-silicon has grain sizes of several tens of nanometers. The mechanism of the amorphization can be explained due to the fact that, during the implantation process, $\mathrm{Ga}^{+}$ion impact can knock $\mathrm{Si}$ atoms from the lattice, resulting in damage in the implanted region of the crystal. If the dose is high enough, the implanted layer will become amorphous. Meanwhile, in the thermal annealing process, silicon atoms can move into lattice 
sites and impurity $\left(\mathrm{Ga}^{+}\right)$can enter substitutional sites in the lattice ${ }^{28}$. We also performed selected area electron diffraction (SAED) measurement of the amorphized and annealed Si films at the points marked inside the blue circles of Fig. 2 (a) and Fig. 2 (b). Based on the SAED data, the diffuse rings indicate that the ion implanted Si was amorphized (Fig. 2 (c)), while the small spots making up a ring from the SEAD of the annealed Si indicate poly crystalline structures, Fig. 2 (d). Another well-known technique used to investigate the crystallinity of materials is the Raman spectroscopy. The Raman spectrum was measured using a laser source with a spot size of $1 \mu \mathrm{m}$, on a released Si frame with a size of approximately $5 \mu \mathrm{m} \times 5 \mu \mathrm{m}$ to avoid the influence of the Si substrate, as shown in the inset of Fig. 2 (e). In the amorphous $\mathrm{Si}$, no Raman peak was observed, while the Raman spectrum of the annealed Si shows a sharp peak at a wave number of approximately $520 \mathrm{~cm}^{-1}$, showing the evidence of the recrystallization due to thermal treatment, Fig. 2 (e). The intensity of the Raman peak at $520 \mathrm{~cm}^{-1}$ of the annealed $\mathrm{Si}$ is smaller than that of pure Si films, indicating that thermal annealing did not fully recover the amorphized Si layer ${ }^{29}$. Therefore, the abovementioned optical measurements show strong evidence of the amorphization and recrystallization in our process.

The concentration of impurity $\left(\mathrm{Ga}^{+}\right)$was characterized using the energy dispersive X-Ray spectroscopy (EDX). The results show that the concentration of $\mathrm{Ga}$ is proportional to the amount of dose. At the dose of $\sim 10^{15} \mathrm{~cm}^{-2}$ used to fabricate the SiNWs in this study, the concentration of Ga was found to be $\sim 2 \mathrm{wt} \%$, corresponding to an atomic concentration of approximately $10^{20} \mathrm{~cm}^{-3}$, which is in agreement with results reported by others previously ${ }^{30}$. The electrical characteristics of the fabricated SiNWs was then performed using a TMHP 4145B analyzer. Experimental data shows that the conductivity of the annealed SiNWs $\left(\sigma \approx 0.2 \mathrm{Scm}^{-1}\right)$ is at least one order of magnitude higher than that of non-annealed SiNWs $\left(\sigma \approx 0.01 \mathrm{Scm}^{-1}\right)$, as shown in Fig. 3. This result indicates that the amorphous SiNWs were recrystallized after the annealing process, and therefore, their carrier mobility and thus their electrical conductivity were enhanced. Additionally, the carrier concentration also increased due to the activation of $\mathrm{Ga}^{+}$by the thermal treatment. The semiconductor type of SiNWs was investigated by applying the back gate voltage $\left(V_{g}\right)$. Under a positive $V_{g}$, the conductance of SiNWs increased, while at a negative $V_{g}$, the conductance of SiNWs decreased. This result indicates that SiNWs is a p-type semiconductor ${ }^{31}$, which is formed by the implantation of $\mathrm{Ga}^{+}$ions into the Si film. The carrier concentration was calculated using the following equation:

$$
N_{p}=\frac{1}{\rho \mu_{p} q}
$$

where $N_{p}$ is the concentration of activated impurity $\left(\mathrm{Ga}^{+}\right) ; \rho$ is the resistivity of the SiNWs; $\mu_{p}\left(100 \mathrm{~cm}^{2} \mathrm{~V}^{-1} \mathrm{~s}^{-1}\right)$ is the mobility of $\mathrm{Ga}^{+}$implanted $\mathrm{Si}$ after annealing ${ }^{30}$; and $\mathrm{q}$ is the

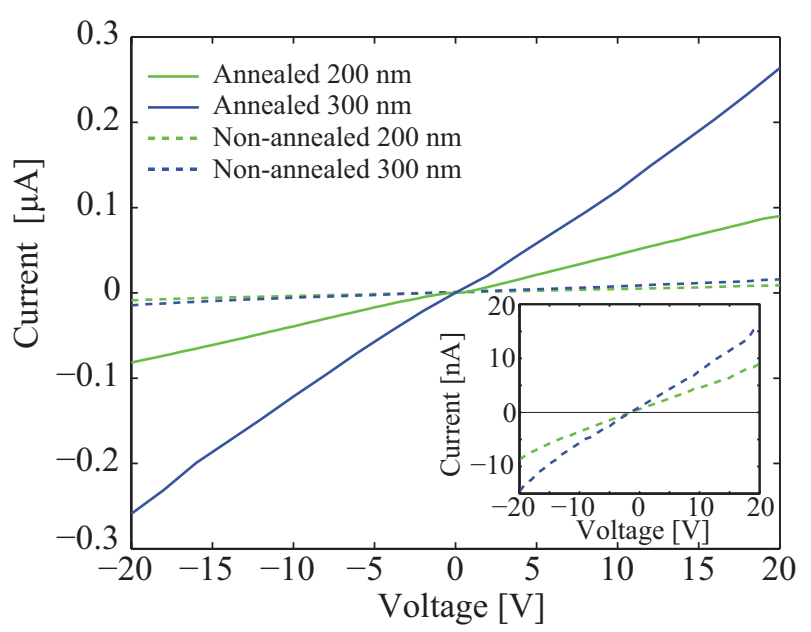

Fig. 3 Comparison of the conductance of annealed/non-annealed SiNWs. Inset: The current-voltage curve of the non-annealed SiNWs plotted in nA scale.

elementary charge $\left(1.602 \times 10^{19} \mathrm{C}\right)$. As a result, the carrier concentration was found to be approximately $2 \times 10^{16} \mathrm{~cm}^{-3}$, which was relatively small in comparison to the dopant concentration $\left(10^{20} \mathrm{~cm}^{-3}\right)$. This result is understandable, since FIB usually creates crystal defects in Si nanostructures, and subsequently carriers can be trapped at the grain boundaries of Si NWs after annealing ${ }^{30}$.

To investigate the piezoresistance of the annealed SiNWs, we applied the bending beam method to induce strain into the SiNWs (Fig. 4 (a) inset). The Si beam with SiNWs on it was clamped at one end, while the other end was applied with different loads. As the distance from the SiNWs to the surface of the Si beam (less than $2 \mu \mathrm{m}$ ) is relatively small in comparison to the thickness of the Si beam ( $200 \mu \mathrm{m})$, we assume that the strain induced into the SiNWs is approximately equal to that of the top surface of the Si beam. Therefore, the applied strain was calculated using the following equation ${ }^{32}$ :

$$
\varepsilon=\frac{M t}{2 E I_{i}}
$$

where $M$ is the bending moment, $t$ is the thickness of the $\mathrm{Si}$ beam, $E$ is is the Young's modulus of $\mathrm{Si}$ in [110] orientation, and $I_{i}$ is the area moment of inertia, respectively. Accordingly, the strain induced into the SiNWs was calculated to be in a range of 0 to $2500 \mathrm{ppm}$. Figure 4 (a) shows the currentvoltage curve of two types of annealed SiNWs with diameters of $200 \mathrm{~nm}$ and $300 \mathrm{~nm}$ under an applied strain of $2500 \mathrm{ppm}$. The decrease in the the current indicates that under an uniaxial tensile strain in [110] orientation, the electrical resistance of both types of the SiNWs increased. Figure 4 (b) presents the relationship between the shift of the current at a constant applied voltage of $20 \mathrm{~V}$ under different strains varied from 0 to $2500 \mathrm{ppm}$, showing a good linear relationship between strain and the change of the current. It is also evident that 


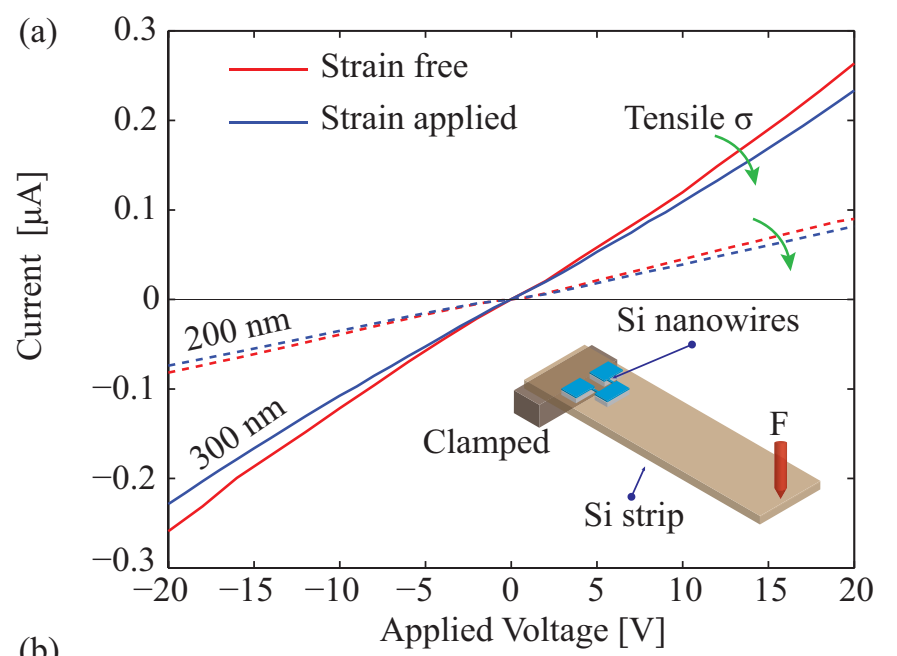

(b)

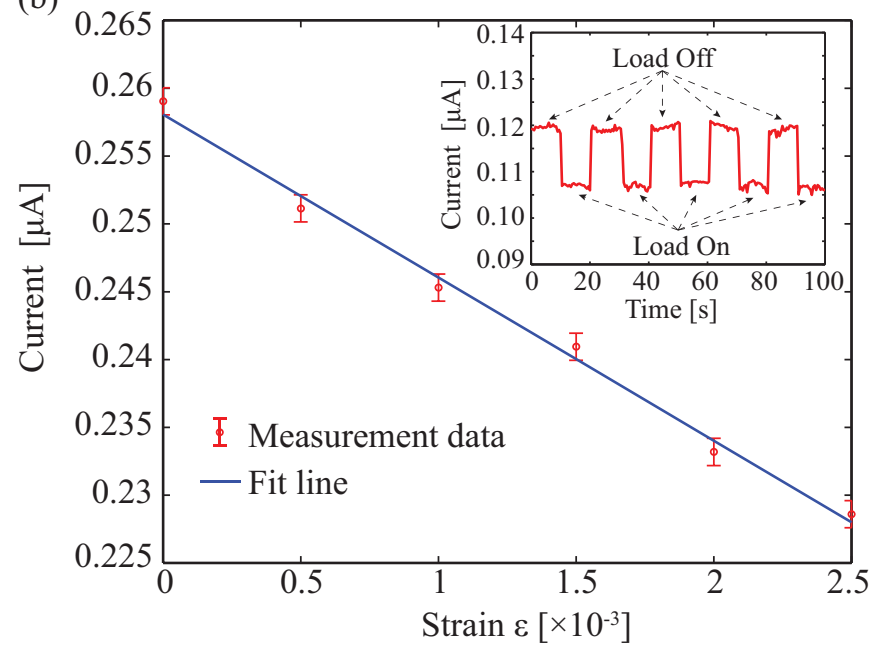

(c) Grain boundary Crystal Si Space charge region

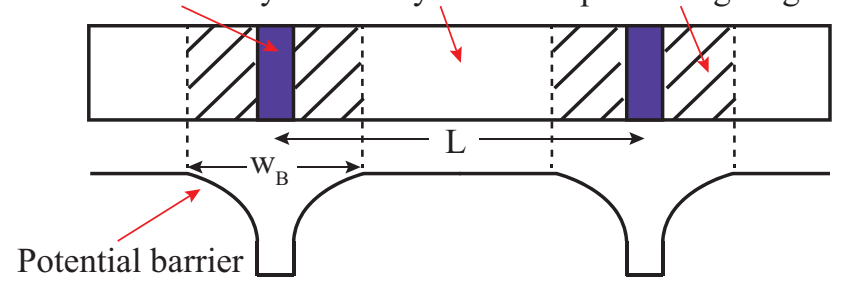

Fig. 4 Characterization of the piezoresistive effect of the annealed SiNWs. (a) The change of current-voltage characteristics of the SiNWs with the widths of $200 \mathrm{~nm}$ and $300 \mathrm{~nm}$ under an applied strain of $2500 \mathrm{ppm}$. Inset: The bending experiment setup; (b) The linear relationship between the current and applied strain of the 300 $\mathrm{nm}$ SiNW at a constant applied voltage of $20 \mathrm{~V}$. Inset: The reproducibility of the current change in the $300 \mathrm{~nm} \mathrm{SiNW}$ after several loading cycles at an applied strain of $2500 \mathrm{ppm}$ and an applied voltage of $10 \mathrm{~V}$. (c) A one dimensional model of the crystalline structure of SiNWs which contains crystalline grains and grain-boundaries.

the piezoresistive effect in the fabricated SiNWs has a good reproducibility after several bending cycles (Fig. 4 (b) inset).
The gauge factor of the annealed SiNWs was then calculated from the ratio of the relative resistance change $(\Delta R / R)$ and the applied strain $(\varepsilon)$ :

$$
G F=\frac{\Delta R / R}{\varepsilon}=-\frac{\Delta I / I}{\varepsilon}
$$

where $I$ is the current of the SiNWs. Consequently, the gauge factor of the $300 \mathrm{~nm}$ was found to be approximately 47, and independent of the applied voltage. Additionally, the gauge factors of the $200 \mathrm{~nm}$ SiNWs $(G F=45)$ and the $300 \mathrm{~nm}$ SiNWs $(G F=47)$ did not show a significant difference. The gauge factor is presented by the change of the geometry and resistivity of the SiNWs ${ }^{2,11}$ :

$$
G F=1+2 \gamma+(\Delta \rho / \rho) / \varepsilon
$$

where $\gamma$ is the Poisson ratio of the SiNWs which varies between 0 and 0.5 . The change of geometry $(1+2 \gamma)$ is between 1 and 2, which is smaller than 5\% of the total gauge factor; therefore, it is obvious that the piezoresistive effect in the annealed SiNWs is due to the change of their electrical resistivity $((\Delta \rho / \rho) / \varepsilon)$. There are various hypotheses developed to explain the piezoresistive effect in SiNWs. He and Yang, and Rowe hypothesized that the potential created at the surface of SiNWs could lead to a piezopinch phenomenon, resulting in a giant piezoresistive effect ${ }^{13,14,20,22}$. However, this electric potential is only significant for SiNWs with a sufficiently small diameter and with a low doping sample $(\rho \approx 10 \Omega \mathrm{cm})$, thus we consider this effect is negligible in our SiNWs ${ }^{20}$. Another possible explanation is the quantum confinement which changes the band energy structure of Si when its size is shrunk down to several nanometers ${ }^{33,34}$. However, again, for SiNWs with a smallest dimension above $60 \mathrm{~nm}$, the influence of quantum confinement is considered to be irrelevant.

Through thermal annealing, amorphous SiNWs are recrystallized, forming poly crystalline SiNWs s0,35,36; therefore, we employ the model of the piezoresistance in poly crystalline materials 37,38 to explain the piezoresistive effect in the annealed SiNWs, as shown in Fig. 4 (c). Accordingly, the resistivity of SiNWs can be expressed by a crystal region and a potential barrier region contribution ${ }^{37,38}$ :

$$
\rho=\rho_{\text {crys }}\left(1-\frac{W_{B}}{L}\right)+\rho_{b} \frac{W_{B}}{L}
$$

where $\rho_{c r y s}$ and $\rho_{b}$ are crystalline $\mathrm{Si}$ and potential barrier resistivity, respectively, while $L$ and $W_{B}$ are the average grain size and the width of the potential barrier. The conductivity of crystalline $\mathrm{Si}\left(\sigma_{\text {crys }}\right)$ and potential barrier $\left(\sigma_{b}\right)$ are given by ${ }^{38,41}$ :

$$
\left\{\begin{array}{l}
\frac{1}{\rho_{\text {crys }}}=\sigma_{\text {crys }}=q^{2} \tau\left(\frac{p_{h}}{m_{h}}+\frac{p_{l}}{m_{l}}\right) \\
\frac{1}{\rho_{b}}=\sigma_{b}=\frac{q^{2} W_{B}}{\sqrt{2 \pi}(k T)^{3 / 2}} \exp \left(-\frac{q V_{B}}{k T}\right)\left(\frac{p_{h}}{\sqrt{m_{h}}}+\frac{p_{l}}{\sqrt{m_{l}}}\right)
\end{array}\right.
$$


here $k, T, V_{B}$ are the Boltzmann constant, the absolute temperature, and the height of potential barrier; $\tau, p$ and $m$ are the relaxation time, the carrier concentration and the effective mass of holes, where the subscripts $h$ and $l$ refer to the heavy hole and light hole in the valence band. Under mechanical strain, the heavy hole and light hole bands are split and warped, leading to the redistribution of holes in these two bands following the fact that holes will fill lower energy levels. Consequently, the re-population of holes results in the change of the hole effective mass. It is obvious that, according to Eq. 6, the hole transfer and mass change phenomenons in the valence band cause the conductivity of both crystalline $\mathrm{Si}$ and potential barrier to change. Utilizing the deformation potential theory into Eq. 6, the gauge factors of low doped ptype crystalline $\mathrm{Si}$ and the potential barrier were numerically calculated to be approximately 160 and 21 , respectively ${ }^{38,39}$. These numerical results are in solid agreement with the experimentally measured GFs of lightly doped single crystalline $\mathrm{Si}^{1,2}$ and poly-silicon ${ }^{37,40}$. Additionally, based on the model shown in Eq. 6, the gauge factor of p-type poly-silicon with its grain sizes varying from several tens to hundreds of nanometers has been estimated to be from 30 to 45 . Therefore, in our annealed SiNWs with the grain size of several tens of nanometers, the gauge factor of 47 indicates that the piezoresistance of the as-fabricated SiNWs can be explained based on the band modification of single crystalline $\mathrm{Si}$ and consequently the change of the thermionic/diffusion currents flowing through grain boundaries under mechanical strain. We also expect that increasing the annealing temperature could enlarge the grain size of poly-silicon and thus could increase its gauge factors. In fact, several studies reported in the literature have proved that thermally annealing the ion implanted Si films at approximately $1000^{\circ} \mathrm{C}$ could almost recover their crystallinity, and as a consequent improving the piezoresistance ${ }^{36,42,43}$. As such, Barlian et al. reported that $\mathrm{B}^{+}$implanted Si films annealed at above $1000^{\circ} \mathrm{C}$ possessed gauge factors of $60 \%$ to $90 \%$ of crystalline $\mathrm{Si}^{43}$. However, $\mathrm{Ga}^{+}$ions which have been commonly deployed in FIB are heavier than $\mathrm{B}^{+}$, causing more crystal damages due to nuclear scattering. Therefore, it is still a challenge to fully recover the $\mathrm{Ga}^{+}$implanted $\mathrm{Si}$ films, and more studies need to be carried out to optimize the annealing conditions.

Finally, we estimate the piezoresistive coefficient of the annealed SiNWs. The longitudinal piezoresistive coefficient of the $\operatorname{SiNWs}\left(\pi_{l}\right)$ is:

$$
\pi_{l}=\frac{\Delta \rho / \rho}{\chi} \approx \frac{G F}{E}
$$

here, $\chi$ is the stress induced to the SiNWs; and E is Young's modulus of the annealed SiNWs. In most previous studies on the piezoresistive effect in SiNWs, the Young's modulus of the SiNWs were assumed to be equal to that of the bulk $\mathrm{Si}$, and there has been no report on the direct measurement of the Young's modulus of SiNWs for calculating the piezoresis- tive coefficient ${ }^{13,15-17}$. As the fabricated SiNWs were amophized and then thermally annealed, it is important to investigate the mechanical properties of SiNWs for estimating the piezoresistive coefficient. Therefore, we applied the following in situ method to measure the Young's modulus of the SiNWs. Annealed SiNWs fabricated by the same process were transferred onto a micro electrostatic comb actuator and fixed by tungsten. The stress applied to the SiNWs was controlled by varying the applied voltage of the comb drive. The deformation of the SiNWs was in situ monitored using SEM as shown in Fig. 5 inset. The detailed description of this experimental setup can be found elsewhere ${ }^{44}$. From the relationship between the applied stress and strain (Fig. 5), the Young's modulus of the annealed SiNWs was calculated to be approximately $170 \mathrm{GPa}$, which is larger than the Young's modulus of amorphized SiNWS, and almost the same as that of bulk single crystalline $\mathrm{Si}$. This result is in agreement with the results obtained from TEM that, after the thermal treatment, the amorphized SiNWs were recrystallized, becoming poly crystalline $\mathrm{Si}$. Consequently, the longitudinal piezoresistive coefficient of the SiNWs was found to be $28 \times 10^{-11} \mathrm{~Pa}^{-1}$.

We also conducted tensile testing on the annealed SiNWs. The results show that in all SiNWs, a brittle fracture occurred within the elastic deformation region, and no yield point was observed. The fractured strength of annealed SiNWs was measured to be approximately $4 \mathrm{GPa}$, indicating that the SiNWs can withstand a large strain of above $2.4 \%$, which is approximately ten times larger than that of the maximum strain applied to the SiNWs in the experiment for characterizing piezoresistive effect shown in Fig. 4.

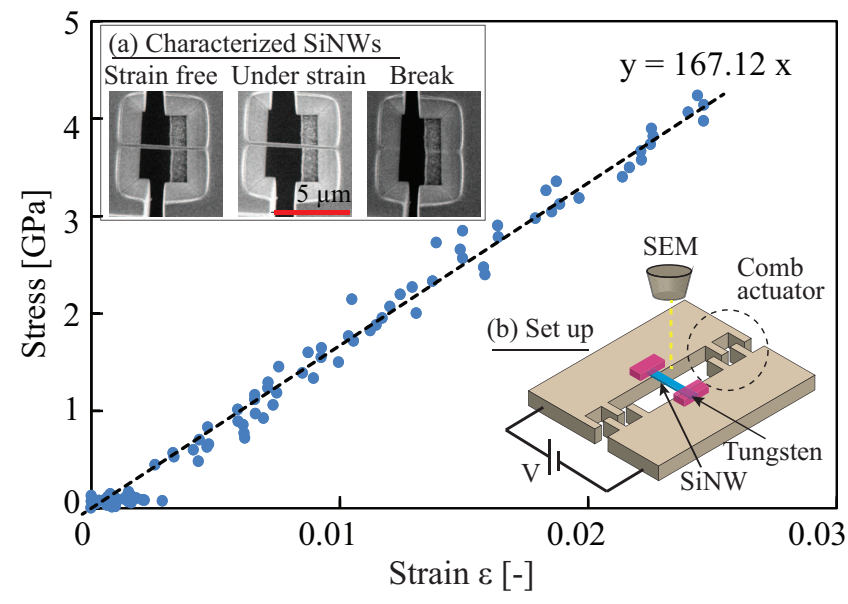

Fig. 5 Characterization of the mechanical property of SiNWs. Inset: (a) The SEM image of the SiNWs with dimensions of $60 \mathrm{~nm} \times 200$ $\mathrm{nm} \times 5 \mu \mathrm{m}$, fabricated using FIB, and then thermally annealed. Two clamping pads were also formed at the ends of SiNWs to ease the consequent transferring and clamping process ${ }^{44}$.;(b) The schematic sketch of the experimental setup, in which the SiNWs were transferred on to an electrostatic comb actuator, and then fixed by tungsten. 
In conclusion, we characterized for the first time the piezoresistive effect in p-type SiNWs fabricated by using the focused ion beam implantation and wet etching process. Gallium ion implantation was carried out to locally amorphize silicon for selective wet etching and for modifying the semiconductor type and doping concentration. After thermally annealing, the electrical conductivity of the SiNWs was enhanced by at least one order of magnitude. The annealed SiNWs possess a large gauge factor of 47 . The compatibility of the FIB process with conventional MEMS fabrications, the advantages of FIB over other EBL/photolithography processes, and the large piezoresistance indicate that the SiNWs fabricated using the method presented in this study are a good candidate for nano electromechanical sensing applications.

This work was performed in part at the Queensland node of the Australian National Fabrication Facility, a company established under the National Collaborative Research Infrastructure Strategy to provide nano and micro-fabrication facilities for Australia's researchers.

\section{References}

1 Smith, C. S., Phys. Rev., 1954, 94, 42.

2 Barlian, A. A.; Park, W. T.; Mallon, J. R. Jr.; Rastegar, A. J.; Pruitt, B. L, Proc. IEEE, 2009, 97(3), 513-552.

3 (a) Phan, H.-P.; Tanner, P.; Dao, D. V.; Wang, L.; Nguyen, N.-T.; Zhu, Y.; Dimitrijev, S., IEEE Electron Device Lett., 2014, 35(3), 399-401. (b) Phan, H.-P.; Dao, D. V.; Tanner, P.; Wang, L.; Nguyen, N.-T.; Zhu, Y.; Dimitrijev, S., Appl. Phys. Lett., 2014, 104, 111905.

4 (a) A. Qamar, H.-P. Phan, D. V. Dao, P. Tanner, T. Dinh, L. Wang, S. Dimitrijev, IEEE Electron Device Lett., 2015, 36(7), 708-710. (b) H.-P. Phan, D. V. Dao, L. Wang, T. Dinh, N.-T. Nguyen, A. Qamar, P. Tanner, S. Dimitrijev, and Y. Zhu, J. Mater. Chem. C, 2015, 3, 1172-1176.

5 Shao, R. W., and K. Zheng, RSC Advances, 2015, 5(43), 34447-34450.

6 Kumar, M., and Bhaskaran, H., Nano Lett., 2015, 15(4), 2562-2567.

7 Tortonese, M.; Barrett, R. C.; Quate, C. F., Appl. Phys. Lett. 1993, 62, 834 .

8 Amarasinghe, R.; Dao, D. V.; Toriyama, T.; Sugiyama, S., 18th IEEE MEMS, Miami, Fl., USA, 2005, 351-354.

9 Yousef, H.; Boukallel, M.; Althoefer, K., Sens. Actuators A, 2011, 167(2), 171-187.

10 Nguyen, M. D.; Phan, H.-P.; Matsumoto, K.; Shimoyama, I., 26th IEEE MEMS, 2013, 617-620.

11 Rowe, A. C. H., J. Mater. Res. 2014, 29(6), 731-744.

12 Dao, D. V.; Nakamura, K.; Bui, T. T.; Sugiyama S., J. Adv. Nat. Sci.: Nanosci. Nanotechnol., 2010, 1(1), 013001.

13 He, R.; Yang, P., Nat. Nanotechnol., 2006, 1, 42.

14 Rowe, A.C.H., Nat. Nanotechnol., 2008, 3, 312.
15 Lugstein, A.; Steinmair, M.; Steiger, A.; Kosina, H.; Bertagnolli, E., Nano Lett., 2010, 10, 3204.

16 Zhang, Y; Liu, X. Y.; Ru, C. H.; Zhang, Y. L.; Dong, L. X.; Sun, Y., J. Microelectromech. Syst., 2011, 20, 959.

17 Milne, J. S., Rowe, A. C. H. ; Arscott, S.; Renner, C., Phys. Rev. Lett., 2010, 105, 226802.

18 Kang, T. K., Nanotechnol., 2012, 23, 475203.

19 Neuzil, P.; Wong, C. C.; Reboud, J., Nano Lett., 2010, 10, 1248.

20 Yang, Y.; Li, X., Nanotechnol., 2011, 22, 015501.

21 S. Zhang, T. Wang, L. Lou, W. M. Tsang, R. Sawada, D.L. Kwong, and C. Lee, J. Microelectromech. Syst., 2014, 23(6), 1396-1407.

22 Rochette, F.; Casse, M.; Mouis, M.; Haziot, A.; Pioger, T.; Ghibaudo, G.; Boulanger, F., Solid-State Electron., 2009, 53, 392.

23 Reyntjens, S.; Puers, R. A., J. Micromech. Microeng., 2001, 11, 287-300.

24 L. A. Giannuzzi, "Introduction to focused ion beams: instrumentation, theory, techniques and practice," Springer Science \& Business Media, 2006.

25 Tseng, A. A., Small, 2005, 1(10), 924939.

26 Fujii, T.; Namazu, T.; Sudoh, K.; Sakakihara, S.; Inoue, S., J. Engineer. Mater. Technol., 2013, 135(4), 041002.

27 M. K. Hatalis, and D. W. Greve, J. Appl. Phys., 1988, 63(7), 2260-2266.

28 R. C. Jaeger, "Introduction to Microelectronic Fabrication: Volume 5 of Modular Series on Solid State Devices (2nd Edition)," Prentice Hall, New Jersey, 2001.

29 A. A. Parr, K. Gill, D. J. Gardiner, J. D. Hoyland, D. Sands, K. Brunson, and R. T. Carline, Semicond. Sci. Technol., 2002, 17(1), 47.

30 Bottger, R.; Bischoff, L.; Schmidt, B.; Krause, M., J. Micromech. Microeng., 2011, 21, 095025.

31 Cui, Y.; Duan, X.; Hu, J.; Lieber, C. M., J. Phys. Chem. B., 2010, 104(22), 5213-5216.

32 (a) Phan, H.-P.; Dao, D. V.; Tanner, P.; Han, J.; Nguyen, N.-T.; Dimitrijev, S.; Walker, G.; Wang, L.; Zhu, Y., J. Mater. Chem. C., 2014, 2, 7176-7179; (b) H.-P. Phan, A. Qamar, D. V. Dao, T. Dinh, L. Wang, J. Han, P. Tanner, S. Dimitrijev, and N.-T. Nguyen, RSC Advances, 2015, 5(69), 56377-56381.

33 Ivanov, T.; Gotszalk, T.; Sulzbach, T.; Rangelow, I., Ultramicroscopy, 2013, 97, 77-84.

34 Nakamura, K.; Dao, D. V.; Isono, Y.; Toriyama, T.; Sugiyama, S. Nanowires, Chapter 15: "Electronic States and Piezoresistivity in Silicon Nanowires", In-Tech, Vienna, Austria., 2010, 297-314.

35 Saxena, S., IEICE Transactions on Electronics, 2010, 93(10), 1495-1498.

36 Xiao, Y. J.; Fang, F. Z.; Xu, Z. W.; Hu, X. T., Appl. Surf. Sci., 2015, 343, 56-69. 
37 Liu, X.; Shi, C.; Chuai, R., Solid State Circuits Technologies, 2010, ISBN: 978-953-307-045-2.

38 Kleimann, P.; Semmache, B.; Le Berre, M.; Barbier, D., Phys. Rev. B: Condens. Matter Mater. Phys., 1998, 57(15), 8966.

39 Toriyama, T.; Sugiyama, S., J. Microelectromech. Syst., 2002, 11(5), 598-604.

40 Schubert, D.; Jenschke, W.; Uhlig, T.; Schmidt, F. M., Sens. Actuator, 1987, 11(2), 145-155.
41 P. J. French, and A. G. R. Evans, "Piezoresistance in polysilicon and its applications to strain gauges," SolidState Electronics, 1989, 32(1), 1-10.

42 J. C. Doll, and B. L. Pruitt, Piezoresistor Design and Applications, Springer, 2013.

43 A. A. Barlian, S. J. Park, V. Mukundan, and B. L. Pruitt, Sensors and Actuators A: Physical, 2007, 134(1), 77-87.

44 Fujii, T.; Sudoh, K.; Sakakihara, S.; Naito, M.; Inoue, S.; Namazu, T., Jpn. J. Appl. Phys., 2013, 52(11R), 110118. 

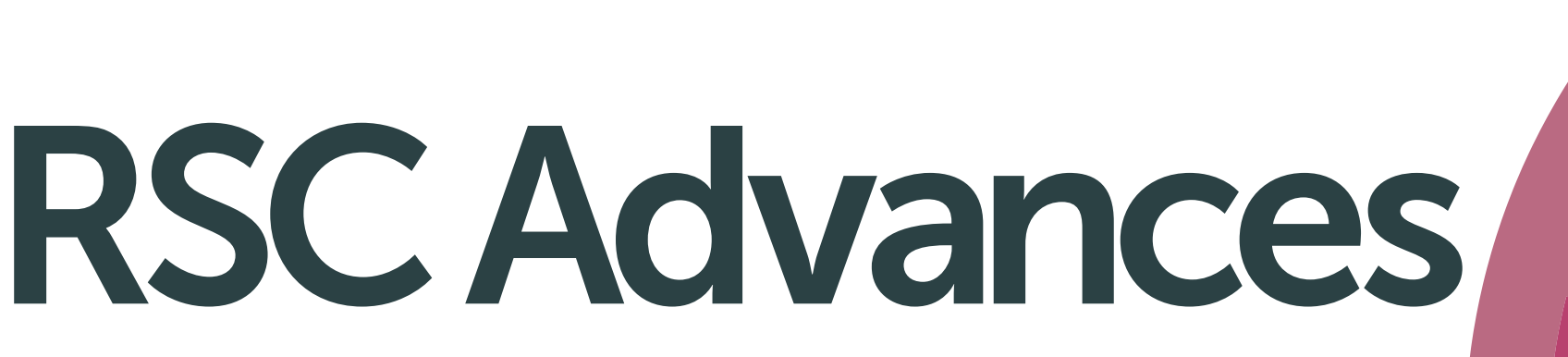

Volume1 Number1 Jan 2013 $\mid$ Pages 1-100

\section{RSC Advances}

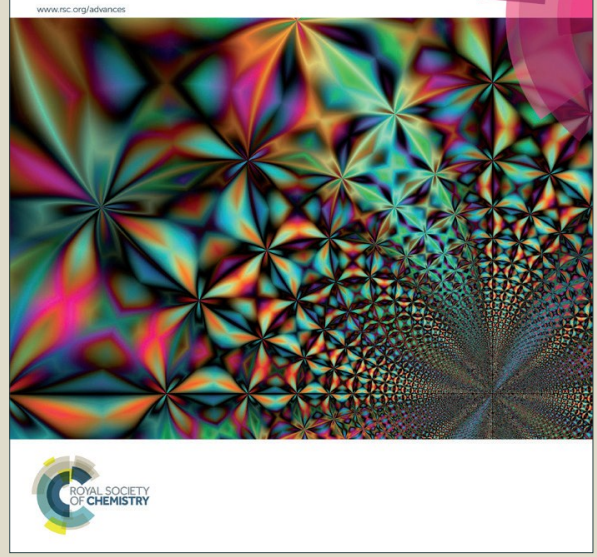

This is an Accepted Manuscript, which has been through the Royal Society of Chemistry peer review process and has been accepted for publication.

Accepted Manuscripts are published online shortly after acceptance, before technical editing, formatting and proof reading. Using this free service, authors can make their results available to the community, in citable form, before we publish the edited article. This Accepted Manuscript will be replaced by the edited, formatted and paginated article as soon as this is available.

You can find more information about Accepted Manuscripts in the Information for Authors.

Please note that technical editing may introduce minor changes to the text and/or graphics, which may alter content. The journal's standard Terms \& Conditions and the Ethical guidelines still apply. In no event shall the Royal Society of Chemistry be held responsible for any errors or omissions in this Accepted Manuscript or any consequences arising from the use of any information it contains. 